\title{
Analytical Modeling of Inactivity Timer in IEEE 802.16m Sleep Mode
}

\author{
Sunggeun Jin, Member, IEEE, Xi Chen, Student Member, IEEE, and Daji Qiao, Member, IEEE
}

\begin{abstract}
IEEE $802.16 \mathrm{~m}$ sleep mode employs a timer in order to determine state transition conditions depending on traffic arrivals destined for a Mobile Station (MS). However, accurate analytical model is not provided yet. We reinvestigate the timer operation and provide an accurate model for further studies.
\end{abstract}

Index Terms-IEEE $802.16 \mathrm{~m}$, power saving, sleep mode.

\section{INTRODUCTION}

I N IEEE $802.16 \mathrm{~m}$ Wireless Metropolitan Area Networks (WMANs), the sleep mode is designed to save energy when a Mobile Station (MS) is serviced with lightly loaded and/or realtime traffic. The $802.16 \mathrm{~m}$ sleep mode has evolved from the 802.16 sleep mode, and hence it shares basic operation regarding listening and sleep windows, where Mobile Station (MS)'s transceiver is activated and inactivated, respectively [1], [2]. Accordingly, many studies have been made for the 802.16 and $802.16 \mathrm{~m}$ sleep mode operations [3], [3]-[18]. However, the $802.16 \mathrm{~m}$ sleep mode has a distinctive feature different from the 802.16 sleep mode. The listening window can be configured to be extended in either case when more packets arrive during ongoing packet transmission time or when an inactivity timer is not expired as elaborated below. When the MS in the listening window completes receptions of the packets buffered at a Base Station (BS), it staying in the listening window may initiate an inactivity timer in order to monitor new packet arrivals. If a new packet arrives prior to the timer's expiration, the MS receives the packet, and thereafter restarts the timer. Otherwise, it goes back to sleeping for energy saving with the sleep window. Therefore, the duration of the listening window increases each time a packet arrives prior to the expiration of (re)started timer. The increment stops when no packet arrives within the timer timeout. We call the time duration, where the MS stays in the listening window, from first starting instant of the timer to expiration instant due to absence of packet arriving, inactivity timer life time.

However, recent works do not provide accurate analytical models for the timer operation [4]-[6]. For example, according to the $802.16 \mathrm{~m}$ standard, when new packet arrives within a timer timeout, the timer should stop to transmit new packet. Only after the transmission, the timer restarts. However, in [4]-[6], the authors assume that the timer does not stop so

Manuscript received December 17, 2011. The associate editor coordinating the review of this letter and approving it for publication was F. Granelli.

S. Jin is with ETRI, Daejeon 305-700, Korea (e-mail: sgjin@etri.re.kr).

X. Chen and D. Qiao are with Iowa State University, Ames, IA 50011, USA (e-mail: \{leon6827, daji\}@iastate.edu).

The research reported in this paper was supported by the KCC (Korea Communications Commission), Korea, under the R\&D program supervised by the KCA (Korea Communications Agency) (KCA-2011-09913-04006), the Information Infrastructure Institute (iCube) of Iowa State University, and the US National Science Foundation under Grant CNS 0831874.

Digital Object Identifier 10.1109/LCOMM.2012.030512.112549

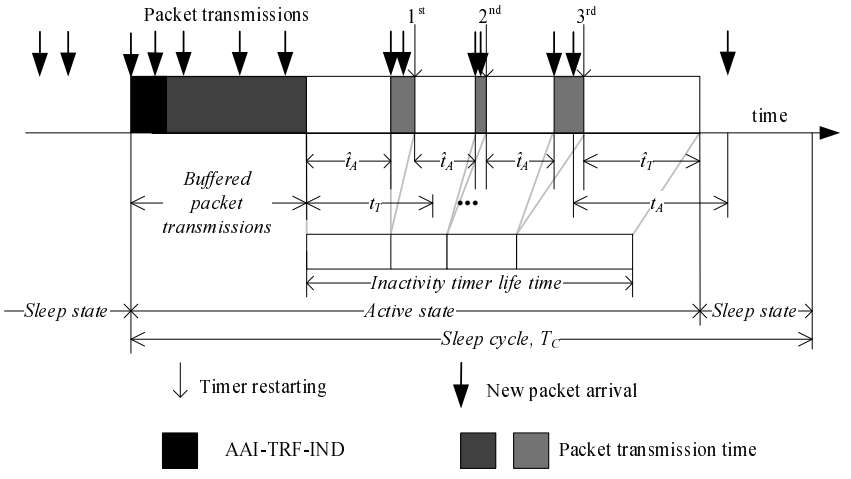

Fig. 1. An example for timer operation in the $802.16 \mathrm{~m}$ WMANs.

that the transmission for the newly arrived packet is deferred until the timer's expiration. This operation is not compatible with the $802.16 \mathrm{~m}$ standard.

In this letter, we first provide accurate analytical model for the inactivity timer life time in the $802.16 \mathrm{~m}$ sleep mode. For simplicity, we only focus on the timer operation in order to rectify analytical models of recent works so that we do not deal with the issues regarding packet transmission delay and power saving efficiency. It implies that we handle the inactivity timer without the packet transmission times since packet transmission times do not influence inactivity timer operations. This letter is organized as follows: in Section II, we explain the timer operation in the $802.16 \mathrm{~m}$ sleep mode. In Section III, we introduce new analytical model for the timer operation. Also, we prove that the analytical model is correctly derived by comparing numerical results with the simulation results. Section IV concludes this letter.

\section{TIMER OPERATION}

The $802.16 \mathrm{~m}$ standard specifies that the sleep mode MS should periodically wake up to monitor new packet arrivals every sleep cycle. The sleep cycle is divided into listening and sleep windows. In the listening window, the MS can receive the traffic indication message, i.e., AAI-TRF-IND, indicating the existence of pending packets destined for the MS. If the indication message indicates the existence of pending packets, the MS stays awake to receive the packets. After completion of the packet receptions, the MS can extend the listening window by starting the timer in order to wait for another packet arriving. The listening window may increase consecutively whenever a new packet arrives within the timer timeout. Upon the timer's expiration, the MS begins the sleep window by inactivating its transceiver. However, the $802.16 \mathrm{~m}$ standard specifies that the listening window cannot increase beyond the sleep cycle. The lengths of listening window, sleep window 
and sleep cycle are negotiated at the beginning of the sleep mode.

Fig. 1 shows an exemplary sleep mode operation with the inactivity timer in the $802.16 \mathrm{~m}$ WMANs. This figure shows that the $802.16 \mathrm{~m}$ BS buffers arriving packets during the sleep window and forwards these packets to the MS during the subsequent listening window. After completion of the buffered packet transmissions, the MS begins the inactivity timer in order to wait for a new packet arrival. In this figure, the listening window extends three times by restarting the inactivity timer, and thereafter the MS enters the sleep window following the listening window upon the expiration of the inactivity timer.

\section{ANALYTICAL MODEL}

For proper analysis, we assume that packet arrival intervals are exponentially distributed with expectation of $1 / \lambda$. The timer's timeout value is assumed to be constant as specified in the $802.16 \mathrm{~m}$ standard. In Fig. 1, random variables $t_{A}$ and $t_{T}$ represent packet arrival interval and timer's timeout value, respectively. Due to the memoryless property of the exponential distribution, it is satisfied that $\operatorname{Pr}\left(t_{A}>t_{b}+t_{T} \mid t_{A}>\right.$ $\left.t_{b}\right)=\operatorname{Pr}\left(t_{A}>t_{T}\right)$, where random variable $t_{b}$ indicates packet transmission time. It implies that inactivity timer life time extends with probability $1-\operatorname{Pr}\left(t_{A}>t_{T}\right)\left(=\operatorname{Pr}\left(t_{A} \leq t_{T}\right)\right)$. For this reason, we denote random variable satisfying $t_{A} \leq t_{T}$ with $\hat{t}_{A}$. Also, we can recognize that $\hat{t}_{A}$ follows a truncated exponential distribution. Similarly, random variable $\hat{t}_{T}$ satisfies the condition that $t_{T}<t_{A}$. We denote the probability density function (pdf) for random variable $t_{A}$ and $t_{T}$ with $f_{A}(t)$ and $f_{T}(t)$, respectively. The timer expires only when packet arrival interval is longer than timer's timeout value. Therefore, the timer expiration probability is derived by:

$$
\begin{aligned}
\operatorname{Pr}\left(t_{A}>t_{T}\right) & =\int_{0}^{\infty} \int_{0}^{t_{A}} f_{A}\left(t_{A}\right) f_{T}(t) d t d t_{A} \\
& =\int_{0}^{\infty} \int_{0}^{t_{A}} \lambda e^{-\lambda t_{A}} f_{T}(t) d t d t_{A} \\
& =\lambda \int_{0}^{\infty} e^{-\lambda t_{A}} \int_{0}^{t_{A}} f_{T}(t) d t d t_{A} \\
& =F_{T}^{*}(\lambda),
\end{aligned}
$$

where $F_{T}^{*}(\lambda)$ is Laplace transform function of pdf $f_{T}(t)$. The timer continues to restart until its expiration. Therefore, we have the probability $\varphi_{i}$ that the timer restarts $i$ times by:

$$
\varphi_{i}=\left(1-F_{T}^{*}(\lambda)\right)^{i} F_{T}^{*}(\lambda)
$$

where $i \geq 0$. From this equation, we can have inactivity timer life time $t_{E}$ by:

$$
t_{E}=\sum_{i=0}^{\infty} \varphi_{i} \sum_{k=1}^{i} \hat{t}_{A}^{(k)}+\hat{t}_{T}
$$

where $\hat{t}_{A}^{(k)}$,s are mutually independent random variables with the same distribution as $\hat{t}_{A}$. A truncated distribution can be denoted by a conditional distribution, and random variable $t_{A}$ is independent to random variable $t_{T}$. Therefore, we have Laplace transform function for random variable $\hat{t}_{A}$ by:

$$
\begin{aligned}
E\left[e^{-s \hat{t}_{A}}\right] & =\int_{0}^{\infty} \int_{0}^{t_{T}} e^{-s \hat{t}_{A}} f_{\hat{t}_{A}}\left(\hat{t}_{A}\right) d \hat{t}_{A} d \hat{t}_{T} \\
& =\int_{0}^{\infty} \int_{0}^{t_{T}} e^{-s t_{A}} f_{t_{A}}\left(t_{A} \mid t_{A} \leq t_{T}\right) d t_{A} d t_{T} \\
& =\frac{1}{1-F_{T}^{*}(\lambda)} \int_{0}^{\infty} \int_{0}^{t_{T}} \lambda e^{-s t_{A}} e^{-\lambda t_{A}} f_{T}\left(t_{T}\right) d t_{A} d t_{T} \\
& =\frac{1}{1-F_{T}^{*}(\lambda)} \int_{0}^{\infty} \frac{\lambda}{s+\lambda} f_{T}\left(t_{T}\right)\left(1-e^{-(\lambda+s) t_{T}}\right) d t_{T} \\
& =\frac{1}{1-F_{T}^{*}(\lambda)} \frac{\lambda}{s+\lambda}\left(1-F_{T}^{*}(s+\lambda)\right) .
\end{aligned}
$$

Similarly, when $t_{T}<t_{A}$, the timer expires. Therefore, we have Laplace transform function for random variable $\hat{t}_{T}$ by:

$$
\begin{aligned}
E\left[e^{-s \hat{t}_{T}}\right] & =\int_{0}^{\infty} e^{-s \hat{t}_{T}} f_{\hat{t}_{T}}\left(\hat{t}_{T}\right) d \hat{t}_{T} \\
& =\int_{0}^{\infty} e^{-s t_{T}} f_{t_{T}}\left(t_{T} \mid t_{T}<t_{A}\right) d t_{T} \\
& =\frac{1}{F_{T}^{*}(\lambda)} \int_{0}^{\infty} \int_{t_{T}}^{\infty} f_{A}\left(t_{A}\right) e^{-s t_{T}} f_{T}\left(t_{T}\right) d t_{A} d t_{T} \\
& =\frac{1}{F_{T}^{*}(\lambda)} \int_{0}^{\infty} e^{-s t_{T}} f_{T}\left(t_{T}\right) \int_{t_{T}}^{\infty} f_{A}\left(t_{A}\right) d t_{A} d t_{T} \\
& =\frac{1}{F_{T}^{*}(\lambda)} \int_{0}^{\infty} e^{-s t_{T}} e^{-\lambda t_{T}} f_{T}\left(t_{T}\right) d t_{T} \\
& =\frac{1}{F_{T}^{*}(\lambda)} F_{T}^{*}(s+\lambda)
\end{aligned}
$$

From Eqs. (2)-(5), we derive Laplace transform function for random variable $t_{E}$ by:

$$
\begin{aligned}
E\left[e^{-s t_{E}}\right]= & E\left[e^{-s \hat{t}_{T}}\right] \sum_{i=0}^{\infty} \varphi_{i}\left(E\left[e^{\hat{t}_{A}}\right]\right)^{i} \\
= & \frac{1}{F_{T}^{*}(\lambda)} F_{T}^{*}(s+\lambda) \sum_{i=0}^{\infty} \varphi_{i}\left(\frac{1}{1-F_{T}^{*}(\lambda)} \frac{\lambda}{s+\lambda}\right. \\
& \left.\times\left(1-F_{T}^{*}(s+\lambda)\right)\right)^{i} \\
= & F_{T}^{*}(s+\lambda) \sum_{i=0}^{\infty}\left(1-F_{T}^{*}(s+\lambda)\right)^{i}\left(\frac{\lambda}{s+\lambda}\right)^{i} \\
= & \frac{1-\left(1-F_{T}^{*}(s+\lambda)\right)}{1-\frac{\lambda}{s+\lambda}\left(1-F_{T}^{*}(s+\lambda)\right)} \\
& \times \sum_{i=0}^{\infty}\left(\frac { \lambda } { s + \lambda } ( 1 - F _ { T } ^ { * } ( s + \lambda ) ) ^ { i } \left(1-\frac{\lambda}{s+\lambda}\right.\right. \\
& \times\left(1-F_{T}^{*}(s+\lambda)\right) \\
= & \frac{F_{T}^{*}(s+\lambda)}{1-\frac{\lambda}{s+\lambda}\left(1-F_{T}^{*}(s+\lambda)\right)} .
\end{aligned}
$$

Herein, we obtain the pdf for random variable $t_{E}$ from Eq. (6), and then apply the pdf to the expectation derivation considering the sleep cycle. The pdf $f_{t_{E}}(t)$ for random vari- 


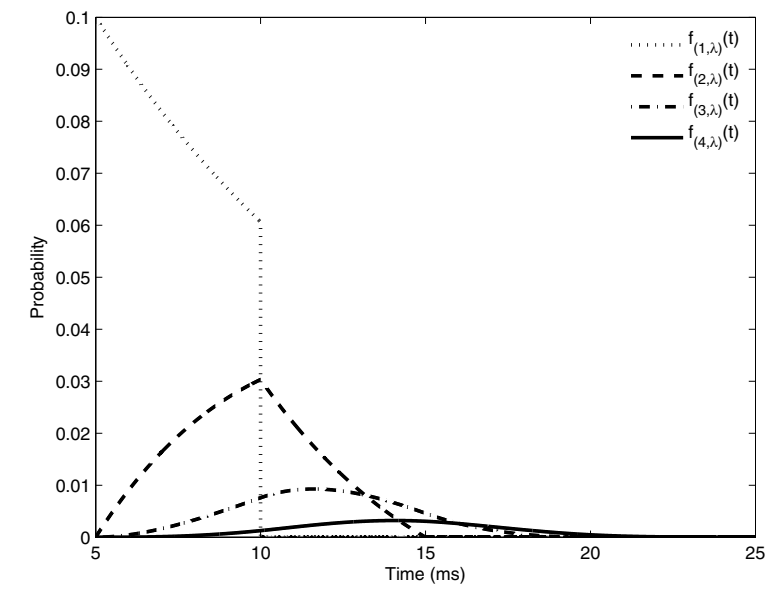

Fig. 2. Probability density function $f_{\gamma(i, \lambda)}(t)$ when $\lambda=0.1, T=5$, and $1 \leq i \leq 4$.

able $t_{E}$ is derived by:

$$
\begin{aligned}
f_{t_{E}}(t)= & \mathcal{L}^{-1}\left\{E\left[e^{-s t_{E}}\right]\right\} \\
= & \sum_{i=0}^{\infty} \mathcal{L}^{-1}\left\{e^{-(s+\lambda) T}\left(\frac{\lambda}{s+\lambda}\right)^{i}\left(1-e^{-(s+\lambda) T}\right)^{i}\right\} \\
= & \sum_{i=0}^{\infty} \mathcal{L}^{-1}\left\{\left(\frac{\lambda}{s+\lambda}\right)^{i}\right. \\
& \left.\times \sum_{j=0}^{i}\left(\begin{array}{l}
i \\
j
\end{array}\right)(-1)^{j} e^{-(s+\lambda) T}\left(e^{-(s+\lambda) T}\right)^{j}\right\} \\
= & \sum_{i=0}^{\infty} e^{-\lambda T} \sum_{j=0}^{i}\left(\begin{array}{l}
i \\
j
\end{array}\right)(-1)^{j}\left(e^{-\lambda T}\right)^{j} \\
& \times f_{\gamma_{(i, \lambda)}}(t-(j+1) T) \mathbf{1}_{t \geq(j+1) T} \\
= & \sum_{i=0}^{\infty} e^{-\lambda T} f_{(i, \lambda)}(t) .
\end{aligned}
$$

Note that we expand this equation by using binomial theorem that $(x+y)^{i}=\sum_{j=0}^{i} x^{j} y^{i-j}$. In this equation, $\gamma_{(i, \lambda)}$ represents random variable following a gamma distribution with expectation $i / \lambda$. Its Laplace transform function $F_{\gamma_{(i, \lambda)}}^{*}(s)=\left(\frac{\lambda}{s+\lambda}\right)^{i}$ and the pdf is denoted with $f_{\gamma_{(i, \lambda)}}(t)$. We use the property of Laplace transform for the derivation of this equation by:

$$
\mathcal{L}^{-1}\left\{F_{\gamma_{(i, \lambda)}^{*}}^{*}(s) e^{-j T s}\right\}=f_{\gamma_{(i, \lambda)}}(t-j T) \mathbf{1}_{t \geq j T} .
$$

For simplicity, we define $f_{(i, \lambda)}(t)=$ $\sum_{j=0}^{i}\left(\begin{array}{c}i \\ j\end{array}\right)(-1)^{j}\left(e^{-\lambda T}\right)^{j} f_{\gamma_{(i, \lambda)}}(t-(j+1) T) \mathbf{1}_{t \geq(j+1) T}$.

Eq. (7) is complete. However, practically this equation requires high computing power for accurate calculations. In order to avoid exhausting computations, we approximate this equation. Fig. 2 shows how $f_{(i, \lambda)}(t)$ varies according to $i$. In this figure, we observe $f_{(i, \lambda)}(t)$ converges to a normal distribution as $i$ increases. The central limit theorem [19] explains sum of independent random variables converges to a normal distribution as illustrated in this figure. For this reason,

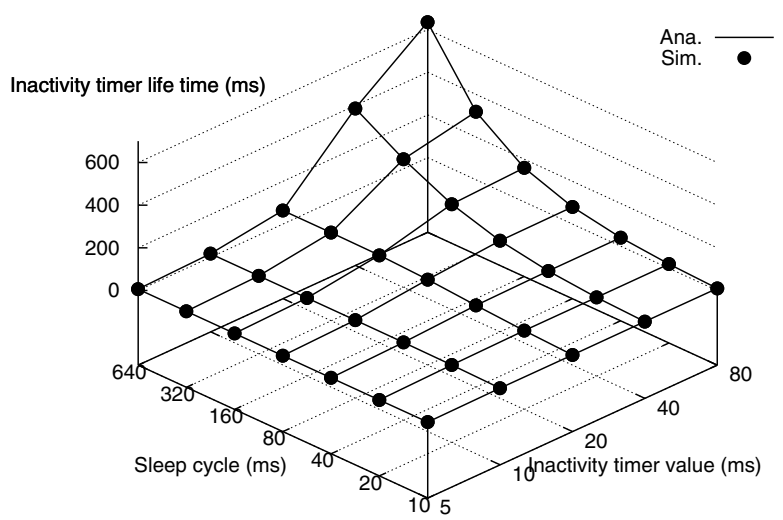

Fig. 3. Inactivity timer life times for the $802.16 \mathrm{~m}$ sleep mode operation when $\lambda=0.1$.

we approximate Eq. (7) by:

$$
f_{t_{E}}(t) \approx \sum_{i=0}^{I-1} f_{(i, \lambda)}(t)+\sum_{i=I}^{\infty} \varphi_{i} f_{\nu(i, \lambda)}(t-T),
$$

where $\nu_{(i, \lambda)}$ is a random variable for normal distribution of which expectation and variance are denoted with $i E\left[\hat{t}_{A}\right]$ and $\operatorname{VAR}\left(\hat{t}_{A}\right)$, respectively. $f_{\nu(i, \lambda)}(t)$ represents pdf for random variable $\nu_{(i, \lambda)} . I$ is an arbitrary number large enough for the central limit theorem. In our evaluations, $I=5 . E\left[\hat{t}_{A}\right]$ and $\operatorname{VAR}\left(\hat{t}_{A}\right)$ are given by:

$$
\begin{aligned}
E\left[\hat{t}_{A}\right] & =\frac{1}{\lambda}\left(\frac{1-(\lambda T+1) e^{-\lambda T}}{1-e^{-\lambda T}}\right), \\
\operatorname{VAR}\left(\hat{t}_{A}\right) & =\frac{2}{\lambda^{2}}\left(\frac{1-\left((\lambda T)^{2} / 2+\lambda T+1\right) e^{-\lambda T}}{1-e^{-\lambda T}}\right) .
\end{aligned}
$$

Finally, we can obtain expected inactivity timer life time for the $802.16 \mathrm{~m}$ WMANs by:

$$
E\left[t_{E}\right]=\int_{0}^{T_{C}} t f_{t_{E}}(t) d t+T_{C}\left(1-\int_{0}^{T_{C}} f_{t_{E}}(t) d t\right),
$$

Fig. 3 illustrates analysis and simulation results. Wellmatched results prove that analytical equations are correctly derived. As shown in this figure, we find that inactivity timer life time increases according to the sleep cycle, inactivity timer value, and packet arrival rate. In this figure, we also observe inactivity timer life times are bounded by sleep cycles. However, we need to obtain exact inactivity timer life times for exact performance evaluations.

\section{CONCLUSION}

We provide an accurate analytical model regarding inactivity timer life time. The proposed equations are expected to be used for further studies relying on numerical analysis of sleep mode operations.

\section{REFERENCES}

[1] IEEE 802.16m, Part 16: Air Interface for Broadband Wireless Access Systems: Advanced Air Interface, May 2011.

[2] IEEE 802.16-2009, Part 16: Air Interface for Broadband Wireless Access Systems, May 2009. 
[3] T.-C. Chen, J.-C. Chen, and Y.-Y. Chen, "Maximizing unavailability interval for energy saving in IEEE 802.16e wireless MANs," IEEE Trans. Mobile Comput., vol. 8, no. 4, pp. 475-487, Apr. 2009.

[4] E. Hwang, K. J. Kim, J. J. Son, and B. D. Choi, "The power-saving mechanism with periodic traffic indications in the IEEE $802.16 \mathrm{e} / \mathrm{m}$," IEEE Trans. Veh. Technol., vol. 59, no. 1, pp. 319-334, Jan. 2010.

[5] — , "The power saving mechanism with binary exponential traffic indications in the IEEE 802.16e/m," Queueing Systems, vol. 62, no. 3, pp. 197-227, July 2009.

[6] S. Baek, J. J. Son, and B. D. Choi, "Performance analysis of sleep mode operation for IEEE $802.16 \mathrm{~m}$ advanced WMAN," in Proc. IEEE ICC'09.

[7] S. Jin, M. Choi, and S. Choi, "Performance analysis of IEEE $802.16 \mathrm{~m}$ sleep mode for heterogeneous traffic," IEEE Commun. Lett., vol. 14, no. 5, pp. 405-407, May 2010.

[8] S. Jin, X. Chen, D. Qiao, and S. Choi, "Adaptive sleep mode management in IEEE $802.16 \mathrm{~m}$ wireless metropolitan area networks," Elsevier Computer Networks, vol. 55, no. 16, pp. 3774-3783, Nov. 2011.

[9] K. De Turck, S. De Vuyst, D. Fiems, S. Wittevrongel, and H. Bruneel, "Performance of the sleep-mode mechanism of the new IEEE 802.16m proposal for correlated downlink traffic," in Proc. NET-COOP'09.

[10] C.-H. Hsu and K.-T. Feng, "A statistical power-saving mechanism for IEEE 802.16 networks," in Proc. IEEE PIMRC'10.
[11] R. K. Kalle, M. Raj, and D. Das, "A novel architecture for IEEE 802.16m subscriber station for joint power saving class management," in Proc. COMSNETS'09.

[12] G. Anastasi, A. Bacioccola, C. Cicconetti, L. Lenzini, E. Mingozzi, and C. Vallati, "Performance evaluation of power management for best effort applications in IEEE 802.16 networks," in Proc. IEEE EW'08.

[13] H.-H. Choi, J.-R. Lee, and D.-H. Cho, "Hybrid power saving mechanism for VoIP services with silence suppression in IEEE 802.16e systems," IEEE Commun. Lett., vol. 11, no. 5, pp. 455-457, May 2007.

[14] K. Han and S. Choi, "Performance analysis of sleep mode operation in IEEE 802.16e mobile broadband wireless access systems," in Proc. IEEE VTC'O6 - Spring.

[15] J. Jang, K. Han, and S. Choi, "Adaptive power saving strategies for IEEE 802.16e mobile broadband wireless access," in Proc. APCC'O6.

[16] L. Kong and D. H. Tsang, "Optimal selection of power saving classes in IEEE 802.16e," in IEEE WCNC'07.

[17] L. Kong, G. K. Wong, and D. H. Tsang, "Performance study and system optimization on sleep mode operation in IEEE 802.16e," IEEE Trans. Wireless Commun., vol. 8, no. 9, pp. 4518-4528, Sep. 2009.

[18] Y. Choi and S. Choi, "Service charge and energy-aware vertical handoff in integrated IEEE 802.16e/802.11 networks," in Proc. IEEE INFOCOM'07.

[19] J. Jacod and A. N. Shiryaev, Limit Theorems for Stochastic Processes. Springer, 1987. 